\title{
On the Interference Channel with Causal Cognition
}

\author{
Martina Cardone ${ }^{\dagger}$, Daniela Tuninetti*, Raymond Knopp ${ }^{\dagger}$, Umer Salim ${ }^{\ddagger}$ \\ ${ }^{\dagger}$ Eurecom, Campus SophiaTech, 450 Route des Chappes, 06410 Biot Email: \{cardone, knopp $\} @$ eurecom.fr \\ ${ }^{*}$ University of Illinois at Chicago, Chicago, IL 60607, USA, Email: danielat@uic.edu \\ $\ddagger$ Intel Mobile Communications Sophia Antipolis, 06560, France, Email: umer.salim@intel.com
}

\begin{abstract}
This paper considers the causal cognitive interference channel that consists of two full-duplex transmitter-receiver pairs sharing the same channel, where one transmitter can causally learn the message of the other transmitter through a noisy link. This channel models unilateral source cooperation. The work focuses on the generalized degrees-of-freedom of the symmetric, i.e. the two interfering links and the two direct links have the same strength, sum-capacity for the Gaussian noise channel. It is shown through evaluation of various achievable schemes that known sum-rate upper-bounds are achievable to within a constant gap regardless of the strength of the channel parameters. The achievable schemes are quite simple in the sense that only superposition coding is used, while it is shown that more complex schemes using binning can achieve a smaller gap.
\end{abstract}

\section{INTRODUCTION}

We consider the Gaussian cognitive overlay system shown in Fig. 1 consisting of two transmitters PTx and CTx and two receivers PRx and CRx. PTx and PRx are so-called primary nodes while CTx and CRx are cognitive nodes. The objectives of the overlay techniques considered here are to firstly allow the cognitive nodes to communicate without hindering the communication of the primary nodes and secondly to enhance the communication reliability of the primary link. To this end, we exploit a lossy communication link between PTx and CTx and we assume that CTx can operate in full-duplex mode on the same communication channel (i.e. same carrier frequency). We treat the case of causal transmission at CTx, in the sense that the knowledge of the primary transmission is only used for encoding at CTx after some processing delay to allow for (partial) decoding or compression of the signal observed at CTx. We shall denote the above described system as Causal Cognitive Interference Channel (CCIC).

Related Work. The presence of a lossy communication link between PTx and CTx enables the CTx to cooperate with the PTx in order to send its message. CTx, in fact, through this noisy channel overhears the signal sent by the PTx and gathers information about its activity. This serves as a basis for unilateral source cooperation, which represents a special case of generalized feedback or bilateral source cooperation [1]. Regarding bilateral source cooperation, the largest known achievable region, to the best of our knowledge, is the one presented in [1]. Here each source splits the message into two sub-messages, i.e. common and private, as in the HanKobayashi's scheme for the non-cooperative IC [2]. Moreover each of these two messages is further sub-divided into a noncooperative and a cooperative part. The former is transmitted as in the classical IC, the latter is delivered to the destination by exploiting the cooperation among the two sources. Bilateral source cooperation has received lots of attention recently. Host-Madsen [3] first studied outer and inner-bounds for the sum capacity of the Gaussian IC with either source or destination bilateral cooperation. Concerning the outer-bound, the author firstly evaluated the cut-set bound, which is easily computable, but turns out to be loose. Consequently, the author tightened the bound by extending to the cooperative case the bounds originally developed by Kramer [4] for the classical IC. Prabhakaran and Viswanath [5] extended the idea in [6, Th.1] and studied an outer-bound for the Gaussian IC with bilateral source cooperation, by treating the noises as independent (this assumption is not without loss of generality for the general cooperative IC). Tuninetti [7] derived a general outer-bound for the IC with bilateral cooperation by extending Kramer's idea [4, Th.1] to general memoryless IC with source cooperation. Concerning unilateral source cooperation, in [8] the authors studied both the cases when the collaborating transmitter works in full-duplex and half-duplex mode. With regard to the full-duplex mode, they developed two achievable schemes: one exploits Partial-Decode-Forward relaying and Gelfand-Pinsker binning and the second extends the first by adding rate splitting and superposition coding. These two schemes can be obtained as special cases of [1].

One of the simplest unilateral source cooperation models is the non-causal cognitive interference channel [9]. This channel is similar to the classical IC with the difference that CTx has a full non-causal knowledge of the primary message. For this channel model the capacity region is exactly known for some parameter regimes and to within 1 bit otherwise [10]. In this paper we remove this ideal assumption by considering the more realistic scenario that CTx learns the message of PTx through a noisy channel. An interesting question we answer in this work is when causal cooperation achieves the same generalized Degrees-of-Freedom (gDoF) as non-causal message knowledge.

Contributions and Paper Outline. This work characterizes the symmetric gDoF of the Gaussian CCIC (G-CCIC), described in Section II. The symmetric sum-capacity is defined to be the maximum sum-rate that PTx and CTx can achieve in the symmetric G-CCIC, i.e., a system with equal interfering links and equal direct links. Our main contribution is twofold (see Section III). First, for the different parameter regimes, we identify simple achievable schemes that are gDoF optimal, from the very general but highly complex scheme of [1]. Then the sum-capacity gap of these gDoF-optimal schemes is shown 


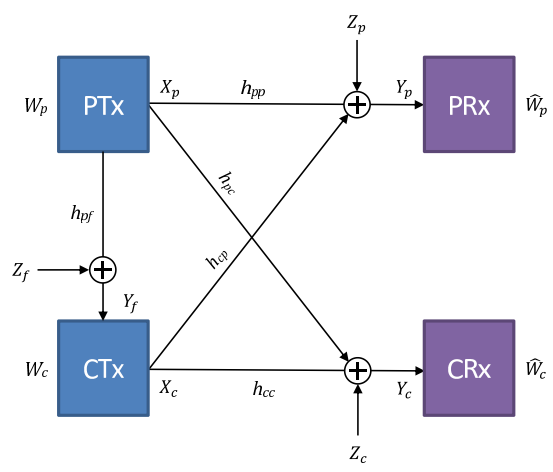

Fig. 1. The 2-user Gaussian Causal Cognitive Interference Channel.

to be bounded by constants in all regimes. These schemes can be used as guidelines to deploy practical cognitive radio systems. Interestingly, the schemes only use superposition coding. It is shown that more complex schemes employing binning / Dirty Paper Coding (DPC) [11] could be used to achieve a smaller gap, thereby pointing to a trade-off between complexity and performance. The proof can be found in Section IV. Secondly, we explicitly identify the parameter regimes where causal cognitive radio offers unbounded gain with respect to the non-cooperative IC and the regimes where it achieves the same $\mathrm{gDoF}$ as non-causal cognition.

\section{System Model AND BACKGROUND}

General Memoryless Channel Model. A CCIC consists of two input alphabets $\left(\mathcal{X}_{p}, \mathcal{X}_{c}\right)$, three output alphabets $\left(\mathcal{Y}_{f}, \mathcal{Y}_{p}, \mathcal{Y}_{c}\right)$ and a memoryless transition probability $\mathcal{P}_{Y_{f}, Y_{p}, Y_{c} \mid X_{p}, X_{c}}$. PTx has a message $W_{p} \in\left[1: 2^{N R_{p}}\right]$ for PRx and CTx has a message $W_{c} \in\left[1: 2^{N R_{c}}\right]$ for CRx, where $N$ denotes the codeword length and $R_{p}$ and $R_{c}$ are the transmission rates for PTx and CTx, respectively, measured in bits per channel use (logarithms are in base 2). The messages $W_{p}$ and $W_{c}$ are independent and uniformly distributed on their respective domains. At time $i \in[1: N]$ the PTx maps its message $W_{p}$ into a channel input symbol $X_{p, i}\left(W_{p}\right)$ and CTx maps its message $W_{c}$ and its past channel observations into a channel input symbol $X_{c, i}\left(W_{c}, Y_{f}^{i-1}\right)$. At time $N$, the PRx outputs an estimate of its intended message $W_{p}$ based on all its channel observations as $\widehat{W}_{p}\left(Y_{p}^{N}\right)$, and similarly CRx provides $\widehat{W}_{c}\left(Y_{c}^{N}\right)$. The capacity region is defined as the convex closure of all non-negative rate pairs $\left(R_{p}, R_{c}\right)$ such that $\max _{u \in\{c, p\}} \mathbb{P}\left[\widehat{W}_{u} \neq W_{u}\right] \rightarrow 0$ as $N \rightarrow \infty$.

Gaussian Noise Channel and gDoF. A single-antenna full-duplex G-CCIC, shown in Fig. 1, is described by the input/output relationship

$$
\left[\begin{array}{c}
Y_{f} \\
Y_{p} \\
Y_{c}
\end{array}\right]=\left[\begin{array}{cc}
h_{p f} & \star \\
h_{p p} & h_{c p} \\
h_{p c} & h_{c c}
\end{array}\right]\left[\begin{array}{c}
X_{p} \\
X_{c}
\end{array}\right]+\left[\begin{array}{c}
Z_{f} \\
Z_{p} \\
Z_{c}
\end{array}\right],
$$

where $\star$ indicates the channel gain that does not affect the capacity region (because CTx can remove its transmit signal $X_{c}$ from its channel output $Y_{f}$ ). The channel gains are complexvalued constants and therefore known to all terminals. The channel inputs are subject to the average power constraints
$\mathbb{E}\left[\left|X_{i}\right|^{2}\right] \leq P_{i} \in \mathbb{R}^{+}, i \in\{p, c\}$. We assume without loss of generality that $Z_{k} \sim \mathcal{C N}(0,1), k \in\{f, p, c\}$. In the following we shall assume that the noises are independent.

An often adopted figure of merit for the Gaussian channel is $\mathrm{gDoF}$ defined as follows. Let $\mathrm{S}>0$ and parameterize

$$
\begin{array}{r}
P_{p}\left|h_{p p}\right|^{2}=P_{c}\left|h_{c c}\right|^{2}:=S=\mathrm{S}^{1}, P_{p}\left|h_{p f}\right|^{2}:=C=\mathrm{S}^{\alpha_{f}}, \\
P_{p}\left|h_{p c}\right|^{2}:=I_{c}=\mathrm{S}^{\alpha_{c}}, P_{c}\left|h_{c p}\right|^{2}:=I_{p}=\mathrm{S}^{\alpha_{p}},
\end{array}
$$

where $\alpha_{c}$ and $\alpha_{p}$ measure the strength of the interference/cross links compared to the direct link, and $\alpha_{f}$ that of the cooperation/feedback link. The gDoF of a Gaussian noise channel is defined as [6], [5]

$$
\mathrm{d}:=\lim _{\mathrm{S} \rightarrow+\infty} \frac{\max \left\{R_{p}+R_{c}\right\}}{2 \log (1+\mathrm{S})}
$$

where the maximization is over all achievable rates $\left(R_{c}, R_{p}\right)$.

In this work we focus on the interference symmetric GCCIC with $\alpha_{c}=\alpha_{p}=\alpha_{i}$. Interference-asymmetric scenarios, with either $\alpha_{c}=0$ or $\alpha_{p}=0$, are studied in [12].

Known Outer-Bounds. In the literature several outerbounds are known for bilateral source cooperation [3], [5], [7]. Here we specialize them for the case of unilateral cooperation on the complex-valued Gaussian channel with independent noises. We define $\mathbb{E}\left[X_{p} X_{c}^{*}\right]=\rho \sqrt{P_{p} P_{c}}$ for some $\rho \in \mathbb{C}$ such that $|\rho| \leq 1$. The outer-bounds we will use are obtained by upper bounding each individual mutual information term over $\rho$ in the bounds in [3], [5], [7]. In particular, the sum-capacity of the G-CCIC can be characterized to within a constant gap by the cut-set bound [3] in (4), the bound from [7] in (5) and the bound from [5] in (6), all given at the top of the next page.

\section{GDOF AND CAPACITY TO WITHIN A CONSTANT GAP}

Our main result is to show that the sum-capacity upper bound in (3) is achievable to within a constant gap for the interference-symmetric G-CCIC. By setting $\alpha_{c}=\alpha_{p}=\alpha_{i}$, the upper bounds in (4), (5) and (6) imply

$$
2 \mathrm{~d} \leq \min \left\{\mathrm{d}^{(\mathrm{CS})}, \mathrm{d}^{(\mathrm{DT})}, \mathrm{d}^{(\mathrm{PV})}\right\},
$$

where

$$
\begin{aligned}
& \mathrm{d}^{(\mathrm{CS})}:=1+\min \left\{\max \left\{1, \alpha_{i}\right\}, \max \left\{1, \alpha_{f}\right\}\right\}, \\
& \mathrm{d}^{(\mathrm{DT})}:=\left[1-\alpha_{i}\right]^{+}+\max \left\{1, \alpha_{i}\right\}, \\
& \mathrm{d}^{(\mathrm{PV})}:=\max \left\{1-\alpha_{i}, \alpha_{i}\right\}+\max \left\{1-\alpha_{i}+\alpha_{f}, \alpha_{i}\right\} .
\end{aligned}
$$

The gDoF upper bound in (7a) has been obtained by applying (2) with the upper bounds in (4), (5) and (6). The details of the proof are omitted for sake of space and can be found in [12].

Fig. 2 shows the gDoF and the gap for the symmetric G-CCIC. The whole set of parameters has been partitioned into multiple sub-regions depending upon different levels of cooperation $\left(\alpha_{f}\right)$ and interference $\left(\alpha_{i}\right)$ strengths. These regimes are numbered from 1 to 6 and the details appear in Section IV. Our main result, proved in Section IV, is 


$$
\begin{aligned}
& \left(R_{p}+R_{c}\right)^{\mathrm{OB}} \leq \min \left\{\left(R_{p}+R_{c}\right)^{(\mathrm{CS})},\left(R_{p}+R_{c}\right)^{(\mathrm{DT})},\left(R_{p}+R_{c}\right)^{(\mathrm{PV})}\right\} \\
& \left(R_{p}+R_{c}\right)^{(\mathrm{CS})} \leq \log (1+S)+\min \left\{\log \left(1+\left(\sqrt{S}+\sqrt{I_{p}}\right)^{2}\right), \log (1+C+S)\right\}, \\
& \left(R_{p}+R_{c}\right)^{(\mathrm{DT})} \leq \min \left\{\log \left(\frac{1+\max \left\{I_{p}, S\right\}}{1+I_{p}}\left(1+\left(\sqrt{S}+\sqrt{I_{p}}\right)^{2}\right)\right), \log \left(\frac{1+C+\max \left\{I_{c}, S\right\}}{1+I_{c}}\left(1+\left(\sqrt{S}+\sqrt{I_{c}}\right)^{2}\right)\right)\right\}, \\
& \left(R_{p}+R_{c}\right)^{(\mathrm{PV})} \leq \log \left(1+\left(\frac{\sqrt{S}}{\sqrt{I_{c}}}+\sqrt{I_{p}}\right)^{2}\right)+\log (1+C)+\log \left(1+\left(\frac{\sqrt{S}}{\sqrt{I_{p}}}+\frac{\sqrt{I_{c}}}{\sqrt{C}}\right)^{2}\right) .
\end{aligned}
$$

Theorem 1 The sum-capacity upper bound in (3) is achievable to within a constant gap of 7.3 bits per channel use regardless of the actual value of the channel parameters.

Before concluding this section, we make some comments on the results we obtained regarding the computation of the gap:

- The gap result of region 6 in Section IV also holds for a big part of region 4 . In particular, it does not apply in the regime $\alpha_{i} \in[2 / 3,1], \alpha_{f}<1-\alpha_{i}$ where the proposed scheme for region 6 does not achieve the optimal $\mathrm{gDoF}$.

- The largest gap in region 6 is 5 bits. This gap may be decreased in several ways. For example, one can optimize the power split between common and private messages instead of using the one presented in [6]. Alternatively, one can develop more complex coding schemes. An example of latter method is represented by the DPC-based achievable scheme that can be found at the end of Section IV. It can be proved [12] that for the regime $\alpha_{i}<1$ and $\alpha_{f}>1$ this DPC-scheme achieves the optimal $\mathrm{gDoF}$ within $2 \mathrm{bits}$, rather than 3 bits as the proposed scheme based on superposition coding only.

- The largest gap occurs when the upper bound in (6) is the tightest. A possible way to reduce the gap would due to develop a tighter upper bound than the one in (6) used here.

- Cooperation does not improve on the gDoF of the classical IC when $\frac{2}{3} \leq \alpha_{i} \leq 2$ or $\alpha_{i} \geq 2, \alpha_{f} \leq 1$ or $\frac{1}{2} \leq \alpha_{i} \leq \frac{2}{3}, \alpha_{f} \leq$ $2 \alpha_{i}-1$. i.e., in these regimes, as far as $\mathrm{gDoF}$ is concerned, there is no need for allowing source cooperation.

- Similarly, cooperation does not improve on the gDoF of the classical relay channel when $1 \leq \alpha_{i} \leq \alpha_{f}$, i.e., in this parameter regime the secondary user acts as a pure relay for the primary user $\left(R_{c}=0\right)$. In other words cognitive radio might not be worth implementing in practical systems.

- Finally, the gDoF of the G-CCIC is equal to that of the non-causal cognitive IC when $\frac{2}{3} \leq \alpha_{i} \leq 2$ or $\alpha_{i} \geq 2, \alpha_{f} \geq$ $\alpha_{i}-1$ or $0 \leq \alpha_{i} \leq \frac{2}{3}, \alpha_{f} \geq \min \left\{1-\alpha_{i}, \alpha_{i}\right\}$, i.e., in these parameter regimes causal cognition attains the ultimate performance limits of non-causal cognition.

\section{Proof}

We define the gap as GAP $=\left(R_{p}+R_{c}\right)^{(\mathrm{OB})}-$ $\left(R_{p}+R_{c}\right)^{(\mathrm{IB})}$. We analyze different regimes:

Region 1: for $\alpha_{i}>2$ and $\alpha_{f} \leq 1$ the tightest upper bound gives $\mathrm{d} \leq 1$. The classical IC in very strong interference with only common messages gives $\left(R_{p}+R_{c}\right)^{(\mathrm{IB})}=2 \log (1+S)$, which using (2) implies $d \geq 1$. This shows the achievability

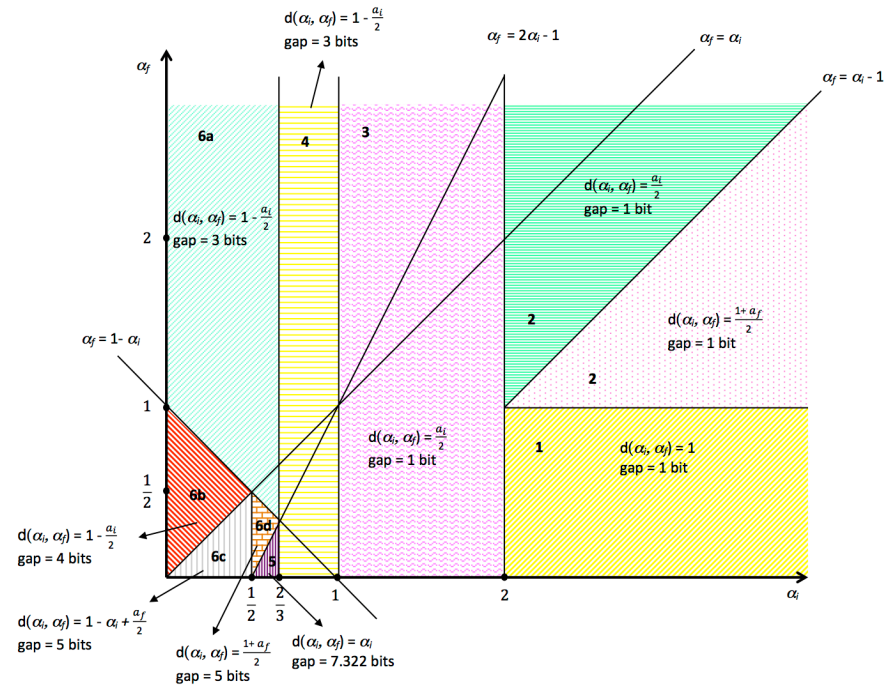

Fig. 2. Optimal gDoF and constant gap for the symmetric G-CCIC in the different regimes of $\left(\alpha_{i}, \alpha_{f}\right)$.

of the gDoF upper bound. From (4) by using $C \leq S$

$$
\mathrm{GAP} \leq \log (1+C+S)-\log (1+S) \leq 1 \text { bit. }
$$

Region 2: for $\alpha_{i}>2$ and $\alpha_{f}>1$ the tightest upper bound gives $\mathrm{d} \leq \frac{1}{2} \min \left\{\alpha_{i}, 1+\alpha_{f}\right\}$. By using [1, Theorem IV.1] with $T_{1}=T_{2}=U_{1}=V_{2}=\emptyset, V_{1}=X_{1}, U_{2}=X_{2}$, and by identifying Node1 with PTx, Node2 with CTx, Node3 with PRx and Node4 with CRx, the following sum-rate is achievable

$$
\begin{gathered}
\left(R_{p}+R_{c}\right)^{(\mathrm{IB})}=\max _{P_{Q} P_{X_{1} \mid Q} P_{X_{2} \mid Q}} \min \left\{I\left(Y_{p} ; Q, X_{c}, X_{p}\right),\right. \\
\left.I\left(Y_{c} ; Q, X_{c}, X_{p}\right), I\left(Y_{f} ; X_{p} \mid X_{c}, Q\right)+I\left(Y_{c} ; X_{c} \mid X_{p}, Q\right)\right\} .
\end{gathered}
$$

In Gaussian noise, we choose $Q, U_{c}, U_{p}$ to be i.i.d. $\mathcal{N}(0,1)$ and define $X_{j}=\sqrt{P_{j}}\left(\beta_{j} Q+\gamma_{j} U_{j}\right):\left|\beta_{j}\right|^{2}+\left|\gamma_{j}\right|^{2} \leq 1, j \in$ $\{p, c\}$. By assuming $\angle h_{c c}-\angle h_{p c}=\angle h_{c p}-\angle h_{p p}$, an achievable sum-rate is

$$
\begin{aligned}
& \left(R_{p}+R_{c}\right)^{(\mathrm{IB})}=\max _{\left(\left|\beta_{c}\right|,\left|\beta_{p}\right|\right) \in[0,1]^{2}} \min \{ \\
& \log \left(1+C\left(1-\left|\beta_{p}\right|^{2}\right)\right)+\log \left(1+S\left(1-\left|\beta_{c}\right|^{2}\right)\right), \\
& \left.\log \left(1+S+I+2 \sqrt{S I}\left|\beta_{p}\right|\left|\beta_{c}\right|\right)\right\} \\
& \geq \begin{cases}\log (1+C)+\log (1+S) & \text { if } C(1+S) \leq I, \\
\log (1+S+I) & \text { if } C(1+S)>I .\end{cases}
\end{aligned}
$$

The pair $\left(\beta_{p}, \beta_{c}\right)=(0,0)$ represents the optimal choice when $C(1+S) \leq I$. The sum-rate corresponding to $C(1+$ 
$S)>I$ has been found by equating the two arguments of the maxmin and by expressing $\beta_{p}$ as a function of $\beta_{c}$. It can be demonstrated that a possibly suboptimal value is $\beta_{c}=0$ that leads to the expression of the sum-rate when $C(1+S)>I$ holds. By using (2), the derived achievable sum-rate implies $\mathrm{d} \geq \frac{1}{2} \min \left\{1+\alpha_{f}, \alpha_{i}\right\}$ since $\alpha_{i}>1$. For the case $S<C \leq I /(1+S)$ using (4) we obtain

$$
\mathrm{GAP} \leq \log (1+C+S)-\log (1+C) \leq 1 \mathrm{bit},
$$

while for $C>I /(1+S)$ using (5) with $S \leq I$ we obtain

$\mathrm{GAP} \leq \log (1+S+I+2 \sqrt{S I})-\log (1+S+I) \leq 1$ bit

since $2 \sqrt{S I} \leq 1+S+I \Longleftrightarrow 0 \leq 1+(\sqrt{S}-\sqrt{I})^{2}$.

Region 3: for $\alpha_{i} \in[1,2]$ the tightest upper bound gives $\mathrm{d} \leq \frac{1}{2} \alpha_{i}$. The classical IC in strong interference with only common messages gives $\left(R_{p}+R_{c}\right)^{(\mathrm{IB})}=\log (1+S+I)$, which using (2) implies $\mathrm{d} \geq \frac{1}{2} \alpha_{i}$ since $\alpha_{i}>1$. From (5) by using $S \leq I$

$\mathrm{GAP} \leq \log (1+S+I+2 \sqrt{S I})-\log (1+S+I) \leq 1$ bit

since $2 \sqrt{S I} \leq 1+S+I \Longleftrightarrow 0 \leq 1+(\sqrt{S}-\sqrt{I})^{2}$.

Region 4: for $\alpha_{i} \in[2 / 3,1)$ the tightest upper bound gives $\mathrm{d} \leq 1-\frac{\alpha_{i}}{2}$. The classical IC with common and private messages and with the power split of [6] gives $\left(R_{p}+R_{c}\right)^{(\mathrm{IB})}=$ $\log (1+S+I)+\log \left(2+\frac{S}{I}\right)-2$, which using (2) implies $\mathrm{d} \geq \frac{2-\alpha_{i}}{2}$ since $\alpha_{i}<1$. From (5) by using $I \leq S$

$$
\mathrm{GAP} \leq \log \left(1+\frac{2 \sqrt{S I}}{1+S+I}\right)+\log \left(\frac{I(1+S)}{S(1+I)}\right)+2 \leq 3
$$

since $2 \sqrt{S I} \leq 1+S+I \Longleftrightarrow 0 \leq 1+(\sqrt{S}-\sqrt{I})^{2}$.

Region 5: $\alpha_{i} \in[1 / 2,2 / 3)$ and $\alpha_{f} \leq 2 \alpha_{i}-1$ in which case the tightest upper bound gives $\mathrm{d} \leq \alpha_{i}$. The classical IC with common and private messages and with the power split of [6] gives $\left(R_{p}+R_{c}\right)^{(\mathrm{IB})}=2 \log \left(1+I+\frac{S}{I}\right)-2$, which using (2) implies $\mathrm{d} \geq \max \left\{\alpha_{i},\left[1-\alpha_{i}\right]^{+}\right\}=\alpha_{i}$ since $\alpha_{i} \in[1 / 2,1]$. From (6)

$$
\begin{aligned}
\mathrm{GAP} & \leq 2 \log \left(1+\left(\frac{\sqrt{S}}{\sqrt{I}}+\sqrt{I}\right)^{2}\right)+\Delta+ \\
& -2 \log \left(1+I+\frac{S}{I}\right)+2 \leq 4+\Delta<7.322 \mathrm{bits}, \\
\Delta:= & \max _{C \in\left[1, I^{2} / S\right]} \log \frac{(1+C)\left(1+\left(\frac{\sqrt{S}}{\sqrt{I}}+\frac{\sqrt{I}}{\sqrt{C}}\right)^{2}\right)}{1+\left(\frac{\sqrt{S}}{\sqrt{I}}+\sqrt{I}\right)^{2}} \leq \log (10) .
\end{aligned}
$$

The upper bound on $\Delta$ is derived as follow: as a function of $C, \Delta$ is a parabola with a minimum in $C$, so its maximum is attained either for $C=1$ or for $C=I^{2} / S$. It can be demonstrated that $\Delta$ is maximum for $C=I^{2} / S$ and here $\Delta=\log (10)$ holds.

Region 6: $\alpha_{i}<2 / 3$ and $\alpha_{f}>\left[2 \alpha_{i}-1\right]^{+}$. We split this region in different sub-regions: Subregion 6a: $1<\alpha_{i}+\alpha_{f}$; here the tightest upper bound gives $d \leq 1-\frac{\alpha_{i}}{2}$. Subregion 6b: $1 \geq \alpha_{f}+\alpha_{i}$ and $\alpha_{f} \geq \alpha_{i}$; here the tightest upper bound gives $\mathrm{d} \leq 1-\frac{\alpha_{i}}{2}$. Subregion 6c: $1>\alpha_{f}+\alpha_{i}, \alpha_{i}<1 / 2$ and $\alpha_{f}<\alpha_{i}$; here the tightest upper bound gives $\mathrm{d} \leq 1-\alpha_{i}+\frac{\alpha_{f}}{2}$.

Subregion 6d: $1 \geq \alpha_{f}+\alpha_{i}, \alpha_{f}<\alpha_{i}$ and $\alpha_{i} \in[1 / 2,1]$; here the tightest upper bound gives $\mathrm{d} \leq \frac{1+\alpha_{f}}{2}$.

By using [1, Theorem 4.1] with $Q=U_{1}=V_{2}=\emptyset$ and by applying Fourier-Motzkin elimination on the resulting region in [1, Theorem 4.1] we get the following achievable sum-rate,

$$
\begin{aligned}
& \left(R_{p}+R_{c}\right)^{(\mathrm{IB})}=\max \min \{ \\
& \min \{[1, e q \cdot(8 a)],[1, e q \cdot(8 b)]\}+[1, e q \cdot(8 d)] \\
& {[1, e q \cdot(8 e)],[1, e q \cdot(8 f)],[1, e q \cdot(8 g)],} \\
& \left.\frac{\min \{[1, e q \cdot(8 a)],[1, e q \cdot(8 b)]\}+[1, e q \cdot(8 \ell)]}{2}\right\},
\end{aligned}
$$

where the maximization is over all joint input distributions $P_{V_{1}, T_{1}, X_{1}, U_{2}, T_{2}, X_{2}}=P_{V_{1}, T_{1}, X_{1}} P_{U_{2}, T_{2}, X_{2}}$.

In Gaussian noise, we choose $V_{1}, T_{1}, U_{2}, T_{2}$ to be i.i.d. $\mathcal{N}(0,1)$ and by identifying Node1 with PTx, Node2 with CTx, Node3 with PRx and Node4 with CRx we let

$$
X_{j}=\sqrt{P_{j}}\left(\beta_{j} U_{j}+\gamma_{j} T_{j}\right):\left|\beta_{j}\right|^{2}+\left|\gamma_{j}\right|^{2} \leq 1, j \in\{p, c\} .
$$

In the following, inspired by the scheme of [6] for the classical IC in weak interference, we set $\left(1-\left|\beta_{c}\right|^{2}\right) I=\left(1-\left|\beta_{p}\right|^{2}\right) I=1$. We do not report here the equations characterizing the achievable region for sake of space. It can be shown, [12, Appendix $\mathrm{G}]$, that the sum-rate in (8) is equal to the term in (8b). Since [1, eq.(8f)] is equal to [1, eq.(8e)] by symmetry, the sum-rate is given by

$$
\begin{aligned}
\left(R_{p}+R_{c}\right)^{(\mathrm{IB})}=\min \{[1, e q \cdot(8 e)],[1, e q \cdot(8 g)]\} \\
=\min \left\{\log \left(\frac{2 I+S}{2 I}\right)+\log \left(\frac{S+I+1}{2}\right),\right. \\
\left.\quad \log \left(\frac{2 I+S}{2 I}\right)+\log \left(\frac{1+C}{I+C}\right)+\log \left(\frac{S+I^{2}+I}{2}\right)\right\} .
\end{aligned}
$$

For future use we note that the second term in the above min is the smallest if $S \geq C(I+1)$. Using (2) the derived achievable rate in $\alpha_{i} \leq 1$ implies that

$$
d \geq \begin{cases}1-\alpha_{i} / 2 & \text { for } 1<\alpha_{i}+\alpha_{f}, \\ 1-\alpha_{i} / 2 & \text { for } 1 \geq \alpha_{i}+\alpha_{f}, \alpha_{f} \geq \alpha_{i}, \\ 1-\alpha_{i}+\alpha_{f} / 2 & \text { for } 1 \geq \alpha_{i}+\alpha_{f}, \alpha_{f}<\alpha_{i}, \alpha_{i}<1 / 2, \\ \left(1+\alpha_{f}\right) / 2 & \text { for } 1 \geq \alpha_{i}+\alpha_{f}, \alpha_{f}<\alpha_{i}, \alpha_{i} \in[1 / 2,1] .\end{cases}
$$

The gDoF upper bound is achievable in $\alpha_{i} \leq 2 / 3$. We analyze separately the different subregimes. For the regimes where $\mathrm{d} \leq$ $1-\alpha_{i} / 2$ we use the upper bound in (5) with $S \geq I$, otherwise we use the upper bound in (6).

\section{Subregion 6a:}

$$
\mathrm{GAP} \leq 2+\log \left(\frac{1+S}{2 I+S} \cdot \frac{I}{1+I}\right)+\log \left(\frac{1+S+I+2 \sqrt{S I}}{S+I+1}\right)
$$

$\leq 3$ bits,

since $\frac{I}{1+I} \leq 1, \frac{1+S}{2 I+S} \leq \max \left\{1, \frac{1}{2 I}\right\}=1$ because $I>1$, $2 \sqrt{S I} \leq 1+S+I \Longleftrightarrow 0 \leq 1+(\sqrt{S}-\sqrt{I})^{2}$ and $I<S$. 


\section{Subregion 6b:}

$$
\begin{aligned}
& \text { GAP } \leq 2+\log \left(\frac{1+S}{1+I}\right)+\log (1+S+I+2 \sqrt{S I})+ \\
& -\log (2 I+S)+\log (I)-\log \left(\frac{1+I}{2 I}\right)-\log \left(S+I^{2}+I\right) \\
& =3+\log \left(\frac{1+S}{2 I+S}\right)+2 \log \left(\frac{I}{1+I}\right)+ \\
& +\log \left(\frac{1+S+I+2 \sqrt{S I}}{S+I^{2}+I}\right) \leq 4 \text { bits, }
\end{aligned}
$$

since $1+S+I<S+I^{2}+I$ and $2 \sqrt{S I}<S+I^{2}+I$.

\section{Subregion 6c:}

$$
\begin{aligned}
& \mathrm{GAP} \leq 2+\log \left(I+S+I^{2}+2 I \sqrt{S}\right)+ \\
& \quad+\log \left(I C+S C+I^{2}+2 I \sqrt{S C}\right)+\log (I+C)+ \\
& \quad-\log (I C)-\log (2 I+S)-\log \left(S+I^{2}+I\right) \\
& \leq 3+\log \left(1+\frac{2 I \sqrt{S}}{S+I^{2}+I}\right)+\log \left(1+\frac{2 I \sqrt{S I}}{2 I^{2}+S I}\right) \leq 5
\end{aligned}
$$

since $2 I \sqrt{S}<S+I^{2}+I$ and $2 I<2 I^{2}+S I$.

Subregion 6d: GAP $\leq 5$ bits, by following exactly the same steps as done for the subregion $6 \mathrm{c}$.

A DPC-based achievable scheme. From [1, Theorem IV.1] with $Q=V_{1}=U_{1}=T_{1}=S_{2}=Z_{2}=V_{2}=U_{2}=\emptyset, Z_{1}=$ $X_{1}$, by identifying Node1 with PTx, Node2 with CTx, Node3 with PRx and Node4 with CRx, and by renaming $S_{1}=Q$, we have

$$
\begin{gathered}
\left(R_{p}+R_{c}\right)^{(\mathrm{IB})}=\max _{P_{Q} P_{X_{p} \mid Q} P_{T_{2}, X_{c} \mid Q}} \min \left\{I\left(X_{p} ; Y_{f} \mid Q, T_{2}, X_{c}\right),\right. \\
\left.I\left(Q, X_{p} ; Y_{p}\right)\right\}+I\left(T_{2} ; Y_{c}\right)-I\left(T_{2} ; Q\right) .
\end{gathered}
$$

In Gaussian noise, we choose $Q, U_{c}, U_{p}$ to be i.i.d. $\mathcal{N}(0,1)$ and define

$$
X_{j}=\sqrt{P_{j}}\left(\beta_{j} Q+\gamma_{j} U_{c}\right):\left|\beta_{j}\right|^{2}+\left|\gamma_{j}\right|^{2} \leq 1, j \in\{c, p\},
$$

and $T_{2}=U_{c}+\lambda_{c} Q$ with $\lambda_{c}$ as in [11] so as to "cancel" $Q$ from $Y_{c}$, that is, so that $I\left(T_{2} ; Y_{c}\right)-I\left(T_{2} ; Q\right)=I\left(T_{2} ; Y_{c} \mid Q\right)$. Then, by assuming $\angle h_{c c}-\angle h_{p c}=\angle h_{c p}-\angle h_{p p}$, an achievable sum-rate is

$$
\begin{aligned}
& \left(R_{p}+R_{c}\right)^{(\mathrm{IB})} \underset{\left(\left|\beta_{c}\right|,\left|\beta_{p}\right|\right) \in[0,1]^{2}}{=} \log \left(1+\frac{\left(1-\left|\beta_{c}\right|^{2}\right) S}{1+\left(1-\left|\beta_{p}\right|^{2}\right) I}\right)+ \\
& \min \left\{\log \left(1+C\left(1-\left|\beta_{p}\right|^{2}\right)\right), \log \left(\frac{1+S+I+2\left|\beta_{c}\right|\left|\beta_{p}\right| \sqrt{S I}}{1+\left(1-\left|\beta_{c}\right|^{2}\right) I}\right)\right\} .
\end{aligned}
$$

Gap for $\alpha_{i}<1$ and $\alpha_{f}>1$ with DPC. By using the upper bound in (5) under the condition $S \geq I$ and the above DPC-based achievable rate, we obtain the following gap

$$
\mathrm{GAP} \leq \log \left(1+\frac{2 \sqrt{S I}}{1+S+2 I}\right)+\log \left(1+\frac{S}{C}\right) \leq 2 \text { bits }
$$

using $0 \leq I$ and $S \leq C$.

\section{CONCLusions}

In this work we studied the CCIC, a network with two source-destination pairs sharing the same channel. In contrast to the classical IC, one transmitter, the CTx, exploits information about the other transmitter, the PTx, from its own channel observations; in contrast to the classical cognitive IC, the CTx learns the message of the PTx in a causal way. We used known outer bounds for bilateral source cooperation by adapting them to the case of unilateral cooperation. Our main contribution consisted in showing that for the interference-symmetric case the sum-capacity of the Gaussian CCIC can be achieved to within a constant gap. Interestingly, the achievable schemes only use superposition coding. It is shown that more complex schemes employing DPC may be used to achieve smaller gaps.

\section{ACKNOWLEDGMENT}

The work of Dr. D. Tuninetti was partially funded by NSF under award number 0643954. The work of Dr. D. Tuninetti was possible thanks to the generous support of TelecomParisTech, Paris France, while the author was on a sabbatical leave at the same institution. Eurecom's research is partially supported by its industrial partners: BMW Group Research \& Technology, IABG, Monaco Telecom, Orange, SAP, SFR, ST Microelectronics, Swisscom and Symantec. The research carried out at Eurecom leading to these results has received funding from the EU Celtic+ Framework Program Project SPECTRA. The research at IMC leading to these results has received funding from the EU FP7 grants SACRA ( $\left.\mathrm{n}^{\circ} 249060\right)$ and iJOIN ( $\left.\mathrm{n}^{\circ} 317941\right)$.

\section{REFERENCES}

[1] S. Yang and D. Tuninetti, "Interference channel with generalized feedback (a.k.a. with source cooperation): Part I: Achievable region," IEEE Trans. on Info. Theory, vol. 57, no. 5, pp. 2686 -2710, May 2011.

[2] T.S. Han and K. Kobayashi, "A new achievable rate region for the interference channel," IEEE Trans. on Info. Theory, vol. 27, no. 1, pp. 49 - 60, Jan. 1981.

[3] A. Host-Madsen, "Capacity bounds for cooperative diversity," IEEE Trans. on Info. Theory, vol. 52, no. 4, pp. $1522-1544$, April 2006.

[4] G. Kramer, "Outer bounds on the capacity of gaussian interference channels," IEEE Transactions on Information Theory, vol. 20, no. 3, pp. 581-586, 2004.

[5] V.M. Prabhakaran and P. Viswanath, "Interference channels with source cooperation," IEEE Trans. on Info. Theory, vol. 57, no. 1, pp. 156 -186, Jan. 2011.

[6] R.H. Etkin, D.N.C. Tse, and W. Wang, "Gaussian interference channel capacity to within one bit," IEEE Trans. on Info. Theory, vol. 54, no. 12, pp. $5534-5562$, Dec. 2008.

[7] D. Tuninetti, "An outer bound region for interference channels with generalized feedback," in Information Theory and Applications Workshop (ITA), 2010, Feb. 2010, pp. 1-5.

[8] Z. Wu and M. Vu, "Partial decode-forward binning schemes for the causal cognitive relay channels," CoRR, vol. abs/1111.3966, 2011.

[9] N. Devroye, P. Mitran, and V. Tarokh, "Achievable rates in cognitive radio channels," IEEE Trans. on Info. Theory, vol. 52, no. 5, pp. 1813 - 1827, May 2006.

[10] S. Rini, D. Tuninetti, and N. Devroye, "Inner and outer bounds for the gaussian cognitive interference channel and new capacity results," IEEE Trans. on Info. Theory, vol. 58, no. 2, pp. 820 -848, Feb. 2012.

[11] M. Costa, "Writing on dirty paper (corresp.)," IEEE Trans. on Info. Theory, vol. 29, no. 3, pp. 439 - 441, May 1983.

[12] M. Cardone, D. Tuninetti, R. Knopp, and U. Salim, "On the gaussian interference channel with causal cognition, or with unilateral source cooperation," arXiv:1207.5319, July 2012. 